\title{
Guest Editorial Foreword to the Special Issue of IHC 2014
}

This special issue of Journal on Interactive Systems is dedicated to Human-Computer Interaction. It contains a selection of five of the best full papers presented at the 13th Brazilian Symposium on Human Factors in Computing Systems (IHC 2014), held in Foz do Iguaçu, from October 27 th to 31 th, 2014. As extended versions of the original conference papers, these works present original content or new contributions when compared to their previous versions and underwent a whole new and independent review process.

IHC 2014 brought the theme "Frontiers of Interaction", aiming to motivate the discussion of ideas and knowledge about diversity in HCI. The theme motivates us to think about the interdisciplinary nature of area. It also refers to the challenges of expanding horizons in order to encompass the diversity of users, respecting their differences and their distinct learning styles. Besides, it has been a great motivation for discussing new forms of interaction that break some of the traditional patterns of knowledge that we were producing. Lastly, the theme also fosters intercultural interaction, reflecting on our knowledge and our function on cultural diversity inherent to HCI phenomena. Thus, papers of this special issue present some interesting discussions about this diversity.

'An Exploratory Qualitative Study on People's Attitudes towards Offline and Online Social Networks' brings a case study conducted by an interdisciplinary team. It explores how Brazilian Facebook users deal with privacy online and off-line, and discuss how these findings may affect HCI design decisions.

In the paper 'Personas-Driven Design for Mental Health Therapeutic Applications', the authors deal with a very relevant challenge, which is the design of applications to support the rehabilitation of chemically dependent and depressive patients. In it, a participatory design process is described, aiming to contribute to good methodological practices in that domain.

The authors of 'Investigating the Case-Based Reasoning Process During HCI Design' turned to case-based reasoning to help identify opportunities to support the design of human-computer interaction. They present an evaluation of the tool they developed - CHIDeK (Computer-Human Interaction Design Knowledge), and its role in motivating the designers' reflective process, triggering their memories of experiences with similar systems, and helping to generate new ideas.
In 'Why and how to investigate interaction design of software development tools', the authors explore the relationship between the usability of software development tools and the quality of end users' interaction with the product these tools contribute to build. As a first step to this still underexplored research problem, they propose an inspection method to characterize communicability and usability aspects of software modeling tools.

'Dynamic and Meta-Context Switching for Gaze-Based Interaction' deals with challenges of gaze-based interaction, proposing a combination of dynamic contextswitching and meta-context switching to reduce the effect of the Midas touch problem and avoiding the difficult fine tuning of dwell-time approaches. The authors conducted two experiments, which provided encouraging results and guidance for future designs and further studies.

We are deeply thankful to all reviewers for their inspiring feedback and recommendations, and to the authors for their hard work and collaboration. Last but not least we would like to thank the editor-in-chief, Alberto Raposo, for his invitation, support and guidance during all the process.

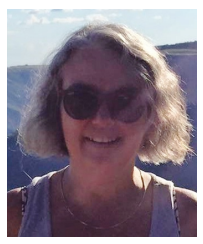

Carla Leitão is senior researcher at Semiotic Engineering Research Group (SERG) at Pontifical Catholic University of Rio de Janeiro (PUC-Rio), since 2002. She has a PhD in Psychology (PUC-Rio) and is the co-author of the books 'Semiotic Engineering Methods for Scientific Research in Human-Computer Interaction' (Morgan \& Claypool Publishers) and 'A Journey Through Cultures: Metaphors for Guiding the Design of CrossCultural Interactive Systems' (Springer Publishing Company).

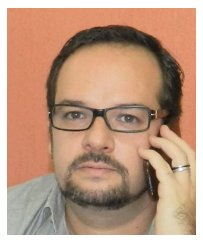

Cristiano Maciel is a researcher at the "Interactive Virtual Environments Laboratory" (LAVI) and a professor in the Institute of Computing, in the Federal University of Mato Grosso - Brazil. He has a $\mathrm{PhD}$ degree in Computation (UFF$\mathrm{RJ}$ ), in collaboration with the Coimbra University (Portugal). At the moment, he is the regional secretary of Mato Grosso in the Brazilian Computer Society (SBC), as well as a member of the Special Commission on HCI and its Executive Council (CEIHC - SBC). His technical interests in Human-Computer Interaction area are mainly related to post-mortem digital legacy, social networks, online education and Web-based interaction. 


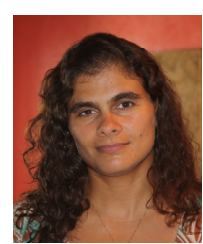

Simone Diniz Junqueira Barbosa is Associate Professor at the Informatics Department of the Pontifical Catholic University of Rio de Janeiro (PUC-Rio), where she teaches, advises and conducts research in the field of Human-Computer Interaction (HCI), mainly as seen from a Semiotic Engineering perspective. Level 2 researcher in $\mathrm{CNPq}$, her current research interests involve: model-based

design of interactive systems; data science and visual analytics; digital storytelling, increasing the quality of use (e.g. usability, communicability, accessibility) of interactive systems in diverse domains, by means of adaptation, analogy-making mechanisms, and other artificial intelligence techniques. In 2010 she co-authored the HCI textbook 'Interação Humano-Computador', for the Brazilian Computer Society series, published by Elsevier. 